\title{
Power Cable Joint Model: Based on Lumped Components and Cascaded Transmission Line Approach
}

\author{
Yan Li, Paul Wagenaars, Peter A.A.F. Wouters, \\ Peter C.J.M. van der Wielen and E. Fred Steennis
}

\begin{abstract}
Models in high frequency range for underground power cable connections are essential for the interpretation of partial discharge (PD) signals arising e.g. diagnostic techniques. This paper focuses on modeling of power cable joints. A lumped parameter odel and a cascaded transmission line model are proposed based on scattering parameters (S -parameters) measurement on a $10 \mathrm{kV}$ oil-filled PILC-PILC straight cable joint in the frequency range of $300 \mathrm{kHz}-800 \mathrm{MHz}$. It is shown that the lumped model is suitable for up to $10 \mathrm{MHz}$ while the transmission line model can cover the whole frequency range. The cascaded transmission line model is applied to simulate the reflection on a $150 \mathrm{kV}$ single core XLPE straight joint. Comparison between measurement and simulation indicates that the model parameters (characteristic impedance and propagation coefficient) can be matched to predict the joint's propagation characteristics.
\end{abstract}

Index Terms: modeling, monitoring, parameter estimation, partial discharges, power cable insulation, transmission lines.

\section{Introduction}

The propagation characteristics of high frequency signals in an underground power cable system are the basis for many power cable diagnostic techniques [1]-[3]. To interpret signals captured at the ends of a cable connection in terms of Partial Discharge (PD) magnitude and propagation time, the propagation path must be accurately modeled. The traveling signal will be affected by components in the cable connection, which consists of cables, cable joints and Ring-Main-Units (RMUs). Each of these components has influence on high frequency signal propagation. For high frequency signals, the underground power cable can be modeled as a transmission line [4]. References [5]-[7] provide a lumped component model for RMU for high frequency phenomena. However, literature on models for a power cable joint for high frequency signals is relatively scarce. The model is dependent on the frequency range of interest. When the partial discharge signal propagates along the cable system for hundreds of meters or more, only frequency components up to $5 \mathrm{MHz}$ [8] remain. On the other hand, if the partial discharge signal arises just aside the joint and it is detected locally at the joint, the frequency of interest can go up to $80 \mathrm{MHz}$ or higher [9], [10].

There are different types of power cable joints, such as straight joint for paper insulated lead covered (PILC) cable, straight joint for cross-linked polyethylene (XLPE) insulated cable, the transition joint, etc. However, they share similar design: a metallic connector to connect the cable cores; insulation material around the connector; a flexible metallic braid to connect the metallic outer layer of the cable on each end. This implies that a generic cable joint model can be designed in which the parameters can be adjusted to match measured behavior.

This paper is organized as follows: Section II gives a brief review on transmission line,

Received: July $26^{\text {th }}, 2012$. Accepted: October $11^{\text {th }}, 2012$ 
Yan Li, et al.

scattering parameters (S-parameters) and [ABCD] matrix, which are used for the present modeling. Section III describes the joint with lumped parameter for frequencies up to a few megahertz and as a cascaded transmission line for frequencies up to hundreds megahertz. In Section IV, S-parameters measurements are presented for a three-core PILC straight joint. The lumped parameter model and the cascaded transmission line model are verified by comparing the modeled S-parameters and the test result in the frequency range of $300 \mathrm{kHz}-800 \mathrm{MHz}$. In Section V, the transmission line model is utilized to model a $150 \mathrm{kV}$ XLPE straight joint's reflection of an injected pulse. Section VI summarizes the conclusions

\section{Theory Review}

This section briefly reviews aspects of transmission line theory and S-parameters to be used for setting up the cable joint model and for comparison with measurements

\section{A. Transmission line theory}

Figure 1 shows the concept of transmission line modeling[11]. It can be characterized by two parameters: the charac-teristic impedance Zc and the propagation coefficient $\gamma$

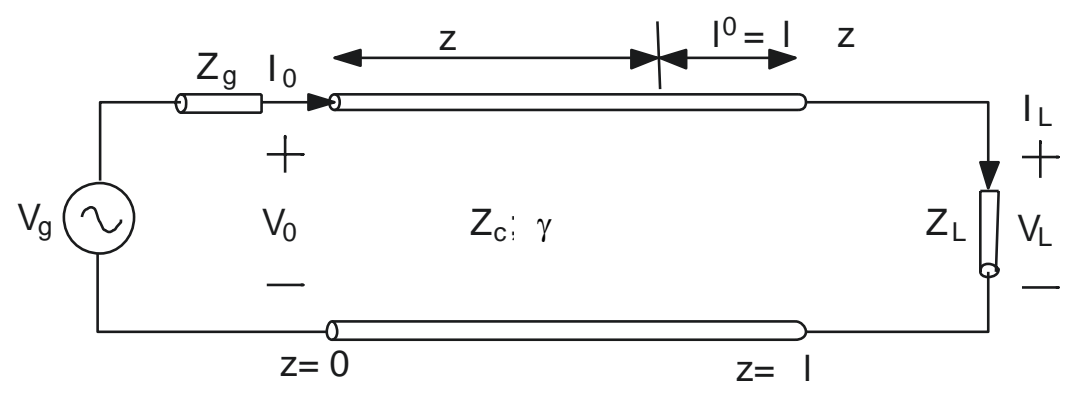

Figure 1. Transmission line model

The voltage and current waves are defined as:

$$
\begin{aligned}
& V_{s}(z)=V_{o}^{+} e^{-\gamma z}+V_{o}^{-\gamma z} \\
& I_{S}(z)=I_{o}^{+} e^{-\gamma z}+I_{o}{ }^{-} e^{\gamma z}=\frac{V_{o}^{+}}{Z_{c}} e^{\gamma z}-\frac{V_{o}^{-}}{Z_{c}} e^{\gamma z}
\end{aligned}
$$

where VS (z) and IS (z) are the voltage and current wave at distance $\mathrm{z}$ from the input.

Substituting the conditions at the input :

$$
\mathrm{Vo}=\mathrm{V}(\mathrm{z}=0), \mathrm{I} 0=\mathrm{I}(\mathrm{z}=0)
$$

into (1) leads to:

$$
V_{L}=\frac{1}{2}\left(V_{o}+Z_{c} I_{o}\right) e^{-\gamma l}+\frac{1}{2}\left(V_{o}-Z_{c} I_{o}\right) e^{\gamma l}
$$




$$
I_{L}=\frac{1}{2 Z_{c}}\left(V_{o}+Z_{c} I_{o}\right) e^{-\gamma l}-\frac{1}{2 Z_{c}}\left(V_{o}-Z_{c} I_{o}\right) e^{\gamma l}
$$

where $l$ is the total length of the transmission line. The voltage reflection coefficient (at the load) $\Gamma L$ is defined as the ratio of the reflected voltage wave to the incident wave at the load, that is :

$$
T_{L}=\frac{Z_{L}-Z_{C}}{Z_{L}+Z_{C}}
$$

The transmitted coefficient is the ratio of the voltage transmission wave to the incident wave, that is:

$$
T_{L}=\frac{2 Z_{L}}{Z_{L}+Z_{C}}
$$

\section{B. S-parameters and $[A B C D]$ matrix}

Linear two-port networks are characterized by a number of equivalent circuit parameters, such as their transfer matrix, impedance matrix, admittance matrix, and scattering matrix. Figure 2 shows a typical two-port network. The matrix elements S11, S12, S21 and S22 are referred to as the scattering parameters or the S-parameters of the device under test (DUT).The parameters S11, S22 have the meaning of reflection coefficients, and S21, S22, the meaning of transmission coefficients. The traveling wave variables a1, b1 at port 1 and a2, b2 at port2 are defined in terms of V1, I1 and V2, I2 and real-valued positive reference impedance Zo as follows:

$$
\begin{aligned}
& a_{1}=\frac{V_{1}+Z_{o} I_{1}}{2 \sqrt{Z_{o}}} \\
& a_{2}=\frac{V_{2}+Z_{0} I_{2}}{2 \sqrt{Z_{o}}} \\
& b 1=\frac{V_{1}-Z_{o} I_{1}}{2 \sqrt{Z_{o}}} \\
& b_{2}=\frac{V_{2}-Z_{o} I_{2}}{2 \sqrt{Z_{o}}}
\end{aligned}
$$

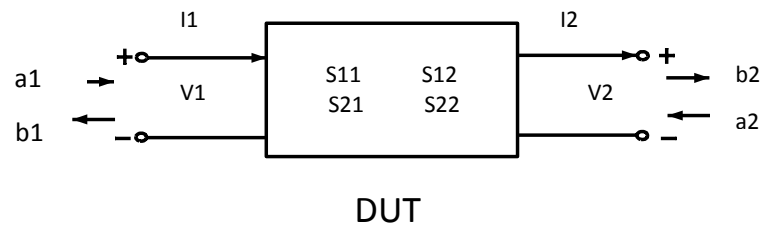

Figure 2. Two-port network

In practice, the reference impedance is chosen to be $Z_{0}=50 \Omega$. Assume that the transmission line in Figure 1 is the DUT, Zo $=\mathrm{Zg}=\mathrm{ZL}$. At lower frequencies the transferand impedance matrices are commonly used, but at microwave frequencies they become difficult to 
measure and therefore, the scattering matrix description is preferred. The S-parameterscan be expressed in terms of transmission line parameters: the characteristic impedance $\mathrm{Zc}$ and the propagation coefficient $\gamma[12]$.

$$
[S]=\frac{1}{D_{s}}\left[\begin{array}{cc}
\left(Z_{0}^{2}-Z_{0}^{2}\right) \sin \gamma & 2 Z_{c} Z_{o} \\
2 Z_{c} Z_{o} & \left(Z_{0}^{2}-Z_{0}^{2}\right) \sin \gamma
\end{array}\right]
$$

where $\mathrm{D}_{\mathrm{S}}=2 \mathrm{Z}_{\mathrm{c}} \mathrm{Z}_{0} \cosh \gamma \mathrm{l}\left(Z_{0}^{2}-Z_{0}^{2}\right) \sin \gamma$. Vice versa, it can be derived from (7) that [13]

$$
\begin{aligned}
& \gamma(\omega)=\frac{1}{l} \cosh ^{-1}\left(\frac{1-S_{11}^{2}+S_{21}^{2}}{2 S_{21}}\right) \\
& Z_{c}(\omega)=Z_{o} \sqrt{\frac{\left(1+S_{11}\right)^{2}-S_{21}^{2}}{\left(1-S_{11}\right)^{2}-S_{21}^{2}}}
\end{aligned}
$$

The input impedance can also be derived from S-parameters:

$$
Z_{\text {in }}=\frac{Z_{0}\left(1+S_{11}\right)}{1-S_{11}}
$$

The disadvantage of S-parameters is that they are not con-venient to be used to model cascaded systems. However, S-parameters can be converted to [ABCD] matrix form which issuitable for cascaded modeling. The $[\mathrm{ABCD}]$ matrix is definedas:

$$
\left[\begin{array}{c}
V_{1} \\
I_{1}
\end{array}\right]=\left[\begin{array}{ll}
A & B \\
C & D
\end{array}\right]\left[\begin{array}{c}
V_{2} \\
I_{2}
\end{array}\right]
$$

Also it can be expressed in terms of S-parameters,

$$
\left[\begin{array}{ll}
A & B \\
C & D
\end{array}\right]=\left[\begin{array}{ll}
\frac{1+S_{11}-S_{22}-d S}{2 S_{21}} & \frac{\left(1+S_{11}+S_{22}+d S\right)}{2 S_{21}} \\
\frac{1-S_{11}-S_{22}+d S}{2 Z_{0} S_{21}} & \frac{1-S_{11}+S_{22}-d S}{2 S_{21}}
\end{array}\right]
$$

where $d S=S_{11} S_{22}-S_{12} S_{21}$, and vice versa:

$$
\left[\begin{array}{ll}
S_{11} & S_{12} \\
S_{21} & S_{22}
\end{array}\right]=\left[\begin{array}{cc}
\frac{Z_{0} A+B-Z_{0}^{2} C-Z_{0} D}{d A} & \frac{2 Z_{0}(A D-B C)}{d A} \\
\frac{2 Z_{0}}{d A} & \frac{-Z_{0} A+B-Z_{0}^{2} C+Z_{0} D}{d A}
\end{array}\right]
$$

where $d A=Z_{0} A+B+Z_{0}^{2} C+Z_{0} D$. For a transmission line the $[A B C D]$ matrix can be expressed as: 


$$
\left[\begin{array}{ll}
A & B \\
C & D
\end{array}\right]=\left[\begin{array}{cc}
\cosh (\gamma) & Z_{c} \sinh (\gamma l) \\
\sinh (\gamma l) / Z_{c} & \cosh (\gamma)
\end{array}\right]
$$

\section{Cable Joint Modeling}

The cable joint used for the experiments is depicted in Figure 3a. The cable oint design is based on an inner and outer joint. The inner joint was made of white BMC polyester (thermohardner). The connectors in the joint were separated by tubes and spacers, both made of PTFE. The space between the inner joint and the cast iron outer joint was filled with 2component polyurethane (brand name Lovinol). The lead sheath of the PILC cables is connected by $50 \mathrm{~mm} 2$ copper. Figure $3 \mathrm{~b}$ shows a schematic drawing of this joint. At both ends of the power cable joint, the cable lead sheath diameter is $48 \mathrm{~mm}$; the cable outer diameter is $62 \mathrm{~mm}$; the joint outer diameter near ends is about $130 \mathrm{~mm}$ and the joint outer diameter at center is approximately $220 \mathrm{~mm}$. The lengths of the PILC cable at both ends are about $14 \mathrm{~cm}$ and $15 \mathrm{~cm}$; while the total length of the joint is about $130 \mathrm{~cm}$. Two approaches are proposed to model this kind of cable joints: one model is based on a lumped parameter description while the other approximates the joint as a cascaded transmission line.

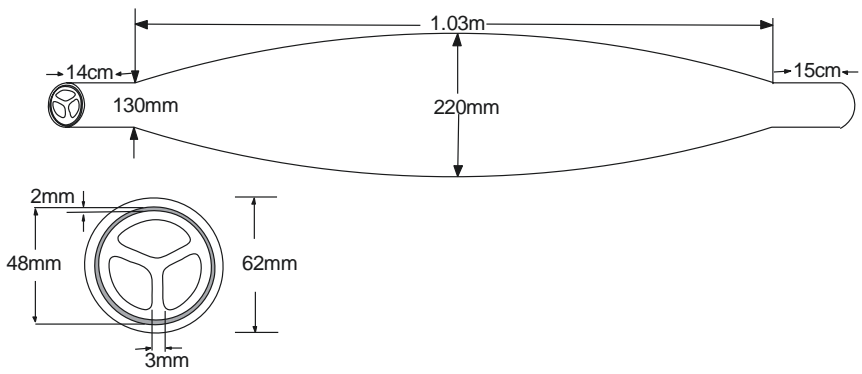

(a) Geometry of the $10 \mathrm{kV}$ oil-filled straight joint.

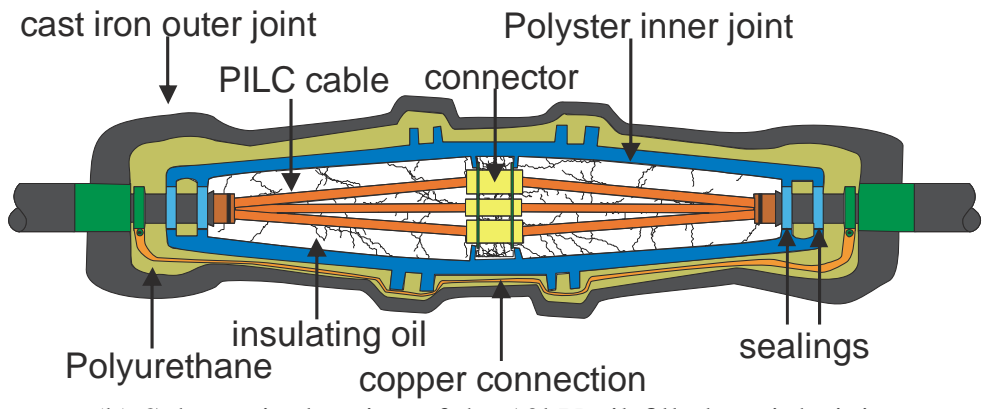

(b) Schematic drawing of the $10 \mathrm{kV}$ oil-filled straight joint

Figure 3. Illustration of DUT.

\section{A. Lumped parameter model}

For low frequency, lumped parameter model can be applied to the power cable joint. This kind of modeling holds for frequencies, where the associated wavelength is at least a factor ten larger than the cable joint length. Good results can be expected up to a frequency of a few tens of megahertz. Here a modified PI model is proposed as shown in Figure 4. 
Yan Li, et al.

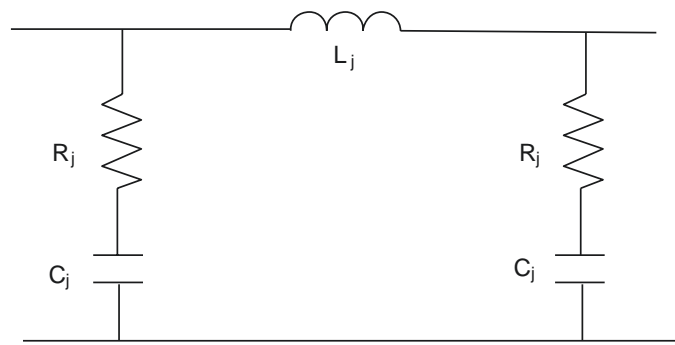

Figure 4. Lumped parameter model for power cable joint

The model consists of L, C and R. The resistance represents the losses in the joint; the inductance and capacitance can produce resonance at certain frequency depending on the geometry and length of the joint. By adjusting the values of the model components the predicted distortion of a signal can be matched to fit with experimental results.

\section{B. Cascaded transmission line model}

The power cable joint has basically a similar structure as a power cable, except that its dimension changes along the length. Because of the inhomogeneous shape of a power cable joint, compared with the power cable, it is non-uniform re-garding to the electric-magmatic field distribution. However, it remains a symmetric and enclosed design. Since the diameter changes relatively slowly over the joint length,a cascaded transmission line is adopted,as shown in Figure 5.

The non-uniform cable joint is divided into m sections Each part is modeled as a separate transmission line with its own characteristic impedance, $\mathrm{Zi}(1 \leq \mathrm{i} \leq \mathrm{m})$ and propagation coefficient, $\gamma \mathrm{i}(1 \leq \mathrm{i} \leq \mathrm{m})$; the length for each section is $\gamma \mathrm{i}$, xi) are dependent on the specific power cable joint geometry and material. If $\mathrm{m}$ increases, the model approximates more closely reality. However, with a higher value of $m$, the computation effort increases and the difference between $\mathrm{Zi}-1, \gamma \mathrm{i}-1$ and $\mathrm{Zi}$, $\gamma \mathrm{i}$ decreases, which means it is more difficult to verify by experiment. So the final model is a balance between accuracy and computational effort.

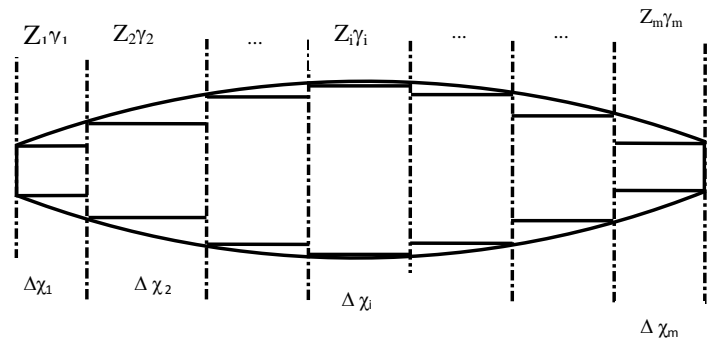

Figure 5. Cascaded model for non-uniform transmission line

The analysis of the proposed cascaded concept shown in Figure 5 is illustrated in Figure 6 . The input and output can be expressed as:

$$
\left[\begin{array}{c}
V_{i n} \\
I_{\text {in }}
\end{array}\right]=\left[\begin{array}{ll}
A_{1} & B_{1} \\
C_{1} & D_{1}
\end{array}\right] \ldots\left[\begin{array}{cc}
A_{i} & B_{i} \\
C_{i} & D_{i}
\end{array}\right] \ldots\left[\begin{array}{ll}
A_{m} & B_{m} \\
C_{m} & D_{m}
\end{array}\right]\left[\begin{array}{c}
V_{m} \\
I_{m}
\end{array}\right]
$$

where the $\mathrm{m}[\mathrm{ABCD}]$ matrixes are corresponding to the $\mathrm{m}$ transmission line segments. 


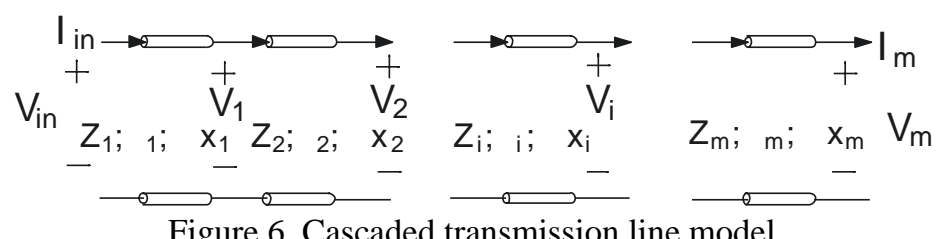

\section{10 KV 3-Core PILC Straight Cable Joint Measurement}

In order to verify the proposed models in section III, the S-parameters of a $10 \mathrm{kV}$ oil-filled 3-core PILC-PILC straight joint are measured and approximated with the models. This section first describes the measurement of the S-parameters and the correction to compensate for the measurement error. Next, both the lumped parameter and the transmission line model are compared with the experimental results.

\section{A. Measurement result and error correction}

A HP 8753C Network Analyzer with HP 85047A S-parameters set is used for the Sparameters test. The vector network analyzer is connected to both sides of the joint under test with a $50 \Omega$ coaxial cable. The test set up is shown in Figure 7. The S-parameters are measured for the cable joint for the frequency range of $300 \mathrm{kHz}-800 \mathrm{MHz}$. As discussed in [7], two propagation modes exist in the three-core power cable, namely shield-to-phase (SP) mode between conductors and earth screen and phase-to-phase (PP) mode between conductors. This paper focuses on the SP mode because it is the detectable signal mode at the earth screen of the cable. This mode is in particular of interest since a PD current sensor can be installed there without safety hazards [14]. Therefore, in the experiment, three cable conductors are connected together. The connection between the network analyzer and the power cable joint is illustrated in Figure 8.

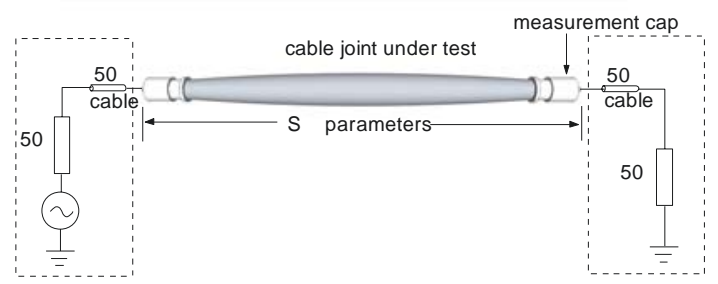

Figure 7. Power cable joint S-parameters measurement system

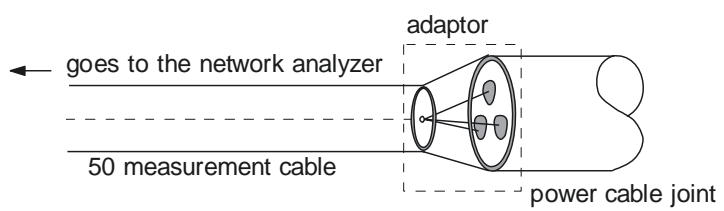

(a) Illustration of the connection between measurement cable to the network analyzer and the DUT

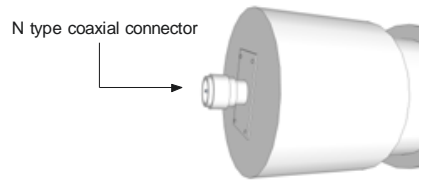

(b) Schematic drawing of the measurement cap (adaptor)

Figure 8. Transitional connection from the coaxial measurement cable to the power cable joint. 
Yan Li, et al.

For the experiment, a $50 \Omega$ coaxial cable is used to connect the network analyzer and DUT; furthermore, an adaptor is needed to connect the coaxial cable and the power cable joint. This coaxial cable and the transitional adaptor connection will distort the measured S-parameters [14]-[16]. According to [16], the effect of the measurement cable is a phase shift in measured S-parameters. If the measured ports are at distance 11,2 from the DUT, the corresponding electrical phase shift for measured S-parameters is $\theta 1,2=\beta 1,211,2$, where $\beta 1,2$ is the phase coefficient (imaginary part of propagation coefficient). Reference [15] pointed out that the combined effect of the measurement cable and the adaptor can be modeled as a piece of lossless transmission line plus a series inductance or a shunt capacitance depending on its influence. This is shown in Figure 9, where $\Delta l_{a d l}$ and $\Delta l_{a d r}$ are the equivalent transmission line additions accounting for the adaptor together with the inductance and capacitance. Here, a shunt capacitance suits the measurement result best. The optimum values for the left measurement cable length and left side capacitance are $4.26 \mathrm{~cm}, 2.36 \mathrm{pF}$ and the right cable length and capacitance are $5.01 \mathrm{~cm}, 9.23 \mathrm{pF}$. These correction parameters are obtained in such a way that maximum repetition of the S-parameters phase from $-\pi$ to $\pi$ is achieved, since the repetition phase indicates a cascaded transmission line configuration. The propagation velocity in the adaptor is taken the same as in the power cable. The obtained lengths of the adaptors are comparable with the physical lengths. It should be noted that the optimized parameters are computer derived and the last two fractional digits are not critical for the further discussion. In fact, If all the four parameters are rounded to one significant figure with the same units, the averaged error is only 8\%. The measured and corrected S-parameters are shown in Figure 10. It can be seen from the comparison that the measurement error is mainly in the phase shift but hardly in the amplitude.

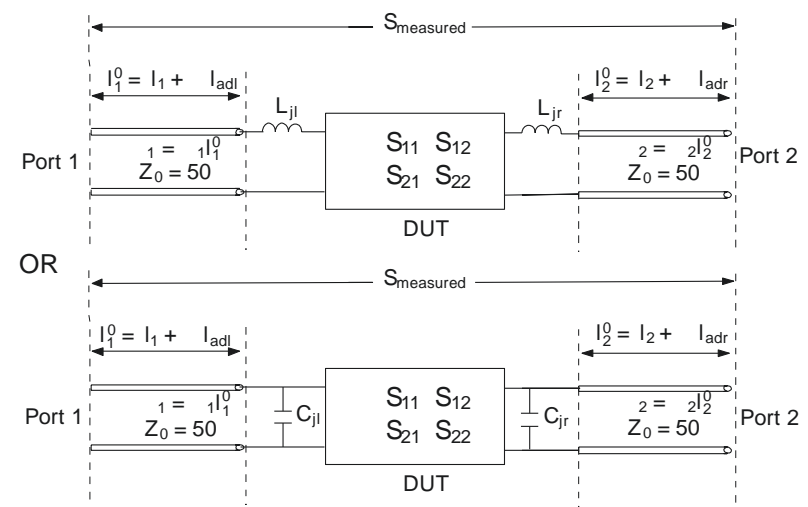

Figure 9. Modeling the combination effect of measurement cable and adaptor

Table 1. Best Fitting Result for cascaded transmisson line model

\begin{tabular}{|c|c|c|c|c|}
\hline$l_{1}(\mathrm{~m})$ & $l_{3}(\mathrm{~m})$ & $\mathrm{Z}_{2}(\Omega)$ & $\gamma_{2}\left(\mathrm{~m}^{-1}\right)$ & $l_{2}(\mathrm{~m})$ \\
\hline 0.35 & 0.34 & 111.4 & $8.6 \alpha+\beta$ & 0.36 \\
\hline
\end{tabular}

\section{B. Model approximation}

Both the lumped parameter model and the cascaded trans- mission line model are adjusted to the measurement result. For the lumped parameter model, $\mathrm{L}, \mathrm{C}$ and $\mathrm{R}$ are optimized for the frequency range $300 \mathrm{kHz}-10 \mathrm{MHz}$. The best fitting parameters are $\mathrm{L}=16.50 \mathrm{nH}, \mathrm{C}=0.40 \mathrm{nF}$ and $\mathrm{R}=6.5 \Omega$. The measured S-parameters and the modeled ones are shown in Figure 11. For the 
cascaded transmission line model, a three cascaded transmission line model is proposed to model the power cable joint under test. The first and third part of the transmission line represent the connected power cable at both ends, while the second transmission line in middle represents the connecting part of the joint, since the most noticeable impedance change appears at the connection point. The characteristic impedance and propagation coefficient of the $10 \mathrm{kV}$ three-phase-PILC power cable are known from previous work, described in [6].
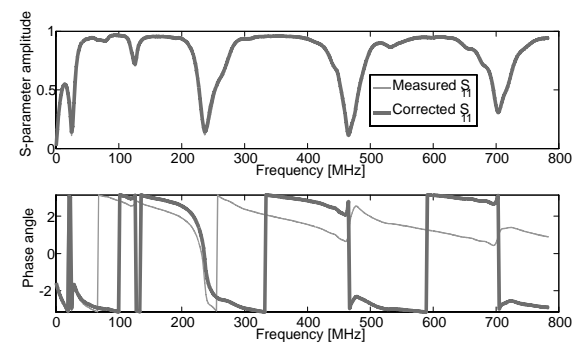

(a) $\mathrm{S}_{11}$ correction
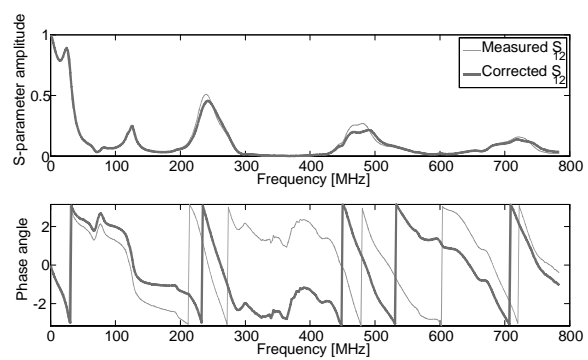

(b) $\mathrm{S}_{12}$ correction
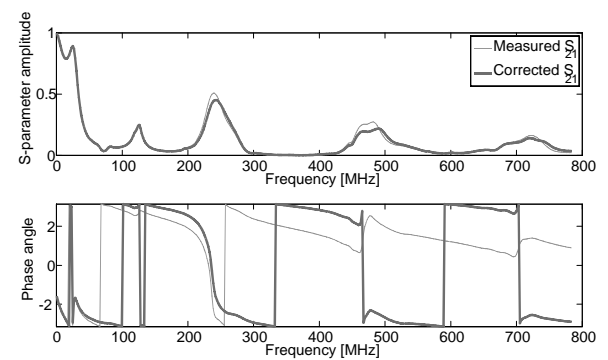

(c) $\mathrm{S}_{21}$ correction
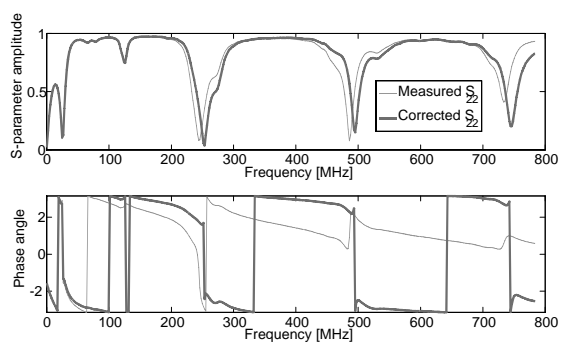

(d) $\mathrm{S}_{22}$ correction

Figure 10. Comparison of the S-parameters before and after correction 
Yan Li, et al.
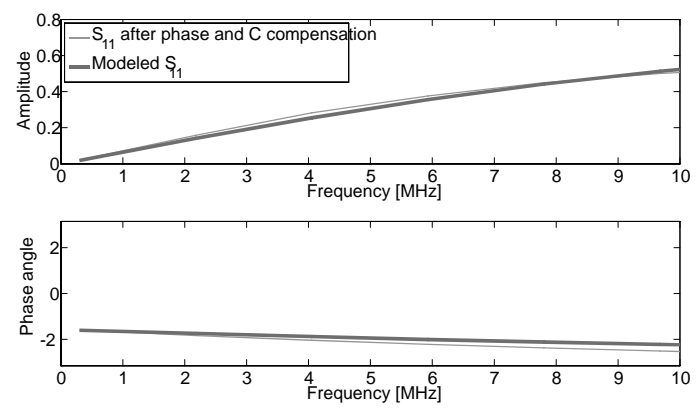

(a) $\mathrm{S}_{11}$ comparison between measurement (after compensation) and model
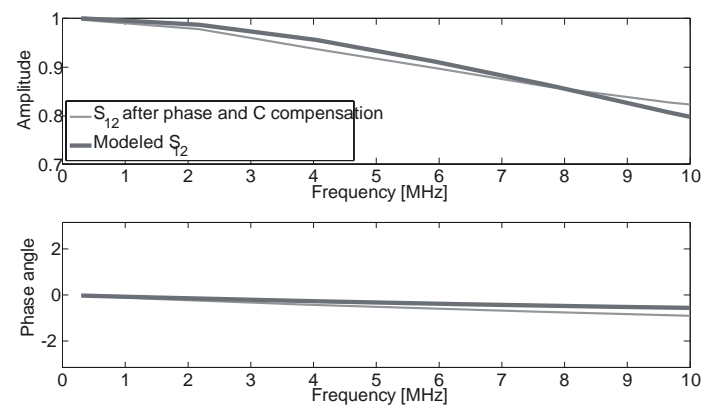

(b) $\mathrm{S}_{12}$ comparison between measurement (after compensation) and model
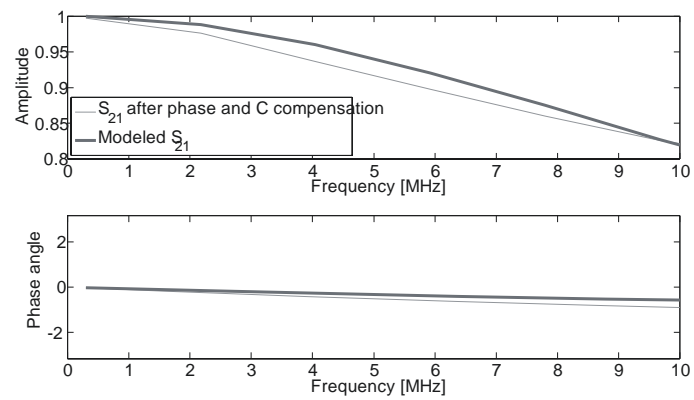

(c) $\mathrm{S}_{21}$ comparison between measurement (after compensation) and model
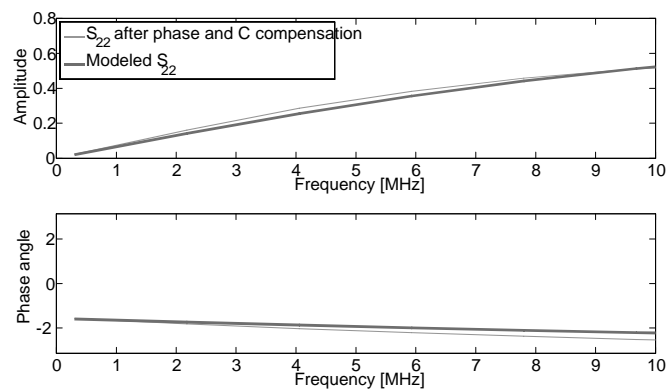

(d) $\mathrm{S}_{22}$ comparison between measurement (after compensation) and model

Figure 11. Comparison between measured S-parameter and modeled ones for lumped model 


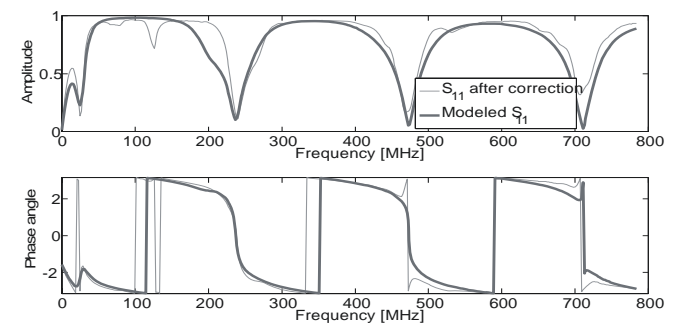

(a) $\mathrm{S}_{11}$ comparison between measurement (after compensation) and model
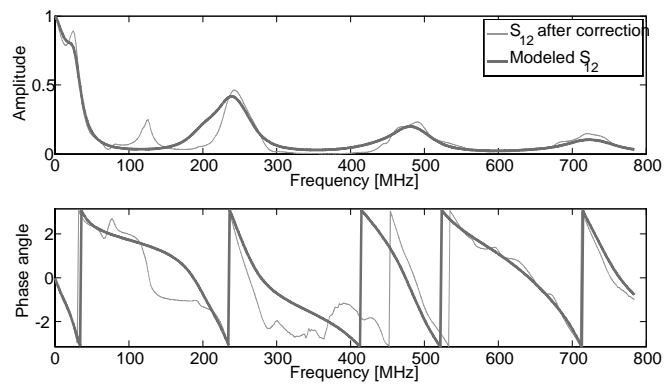

(b) $\mathrm{S}_{12}$ comparison between measurement (after compensation) and model
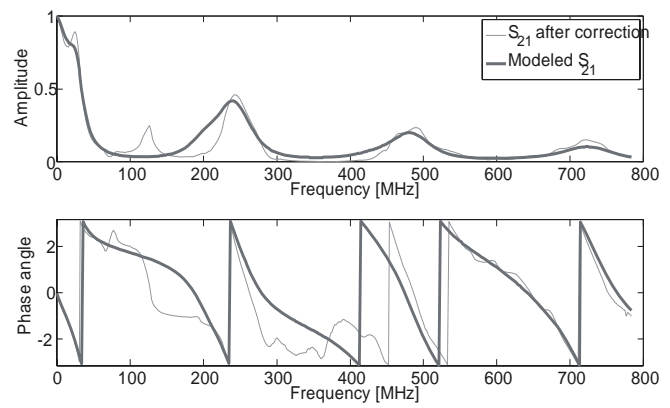

(c) $\mathrm{S}_{21}$ comparison between measurement (after compensation) and model
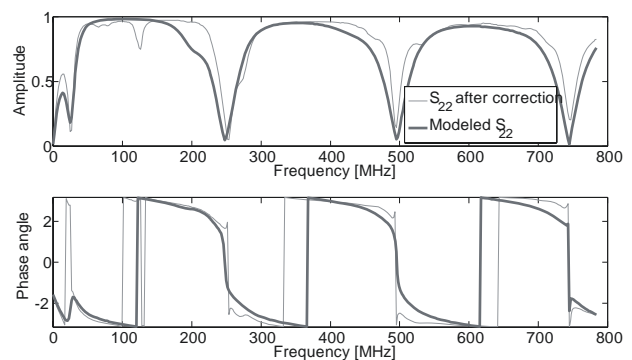

(d). $\mathrm{S}_{22}$ comparison between measurement (after compensation) and model

Figure 12. Comparison between measured S-parameters and modeled values for the cascaded transmission line model 
Yan Li, et al.

The characteristic impedance is $10.0 \Omega(\mathrm{Z} 1=\mathrm{Z} 3=\mathrm{Zc})$ and the frequency dependent propagation coefficient $(\gamma 1=\gamma 3=\gamma)$ are therefore already determined. Only the lengths of end segments 11,13 and the middle segment transmission line parameters Z2, $\gamma 2$, 12 are still to be determined. The best fitting results are shown in Table I. The section length and impedance are chosen such that the model can match the measured S-parameters. Concerning the propagation constant, since the insulation material permittivity for the power cable joint does not differ too much from the power cable, the phase coefficient $(\beta)$ is taken equal to the value for the cable. For the attenuation, the relatively thicker insulation layer radius is expected to increase the losses. The attenuation constant $\alpha$ given in Table I is modified for the joint compared to the cable by a multiplicative factor.

The modeled S-parameters and the measured values are shown in Figure 12. There is an artifact in all measured S-parameters around $125 \mathrm{MHz}$, which might be caused by the network analyzer [15]. Based on the above analysis, it can be concluded that the lumped parameter model can fit the measurement result up to $10 \mathrm{MHz}$ while the cascaded transmission line model can cover the frequency range from $300 \mathrm{kHz}$ to $800 \mathrm{MHz}$. The cascaded transmission line model is wider applicable and therefore the utilization of the cable joint model will focus on this approach.

\section{Model Application}

In this section, the transmission line model is applied to model a straight joint reflection $1103 \mathrm{~m}$ away from the injected pulse at open end of a $150 \mathrm{kV}$ XLPE cable. For the measurement, a pulse is injected into the open end. At distance of $1103 \mathrm{~m}$ locates the straight joint for the XLPE cable. The injected pulse will be reflected at this point and measured at the injection end. The principle is shown in Figure 13a. The function generator is connected via 50 $\Omega$ coaxial cable to both the oscilloscope and the power cable.

The oscilloscope is placed close to the function generator and the distance from the generator to the power cable is about $47 \mathrm{~m}$. An adaptor is used to connect the coaxial cable and the power cable. The measured injected pulse and reflected pulse are shown in Figure 14a. The first reflection after the injection is the pulse reflected from the adaptor while the second reflection is from the straight joint. The characteristic impedance $\mathrm{Zc}$ and propagation constant $\gamma \mathrm{c}$ of the $150 \mathrm{kV}$ cable are measured. The impedance is $25.8 \Omega$ while the propagation velocity is $186 \mathrm{~m} / \mu \mathrm{s}$. Also the measurement coaxial cable and the adaptor are calibrated as discussed in [7].

The cable system can be described with $[\mathrm{ABCD}]$ matrix as:

$$
\begin{aligned}
{\left[\begin{array}{ll}
A & B \\
C & D
\end{array}\right]=} & {\left[\begin{array}{ll}
A_{m} & B_{m} \\
C_{m} & D_{m}
\end{array}\right]\left[\begin{array}{ll}
A_{a d} & B_{a d} \\
C_{a d} & D_{a d}
\end{array}\right] } \\
& {\left[\begin{array}{ll}
A_{c 1} & B_{c 1} \\
C_{c 1} & D_{c 1}
\end{array}\right]\left[\begin{array}{ll}
A_{j} & B_{j} \\
C_{j} & D_{j}
\end{array}\right]\left[\begin{array}{ll}
A_{c 2} & B_{c 2} \\
C_{c 2} & D_{c 2}
\end{array}\right] }
\end{aligned}
$$

where the subscript $\mathrm{m}$ indicates the $50 \Omega$ measurement cable, "ad" indicates the adaptor, "c1" means the first piece of the power cable, "j" means joint, "c2" refers to the second piece of the power cable. The coaxial measurement cable and the power cable are treated as transmission 
lines and the $[A B C D]$ matrix can be derived as in (13) using the measured results. The adaptor is regarded as a series impedance. Since it is calibrated during the measurement, its impedance is known and can be written in terms of the $[A B C D]$ matrix:

$$
\left[\begin{array}{cc}
A_{a d} & B_{a d} \\
C_{a d} & D_{a d}
\end{array}\right]=\left[\begin{array}{cc}
1 & Z_{a d} \\
0 & 1
\end{array}\right]
$$

According to (9) and (12), the input impedance of the cable system can be derived. In order to simulate the reflections from the injection in the cable system, the cable system needs to be connected to an ideal voltage source (zero internal impedance). The voltage source provides a pulse signal injected in the cable system. The schematic simulation set up is shown in Figure $13 \mathrm{~b}$. Here, the injected pulse is loaded with the cable system in series with a $50 \Omega$ impedance. Vmeasure is the point where voltage is extracted to compare with field measurement. The $50 \Omega$ impedance matches the measurement cable's impedance to avoid reflections from the injection end between the source and the measurement cable. The injected and reflected pulse can be obtained from:

$$
I_{i n j}+I_{r e f}=\frac{V_{i n j}}{Z_{i n}+50}
$$

The only undetermined parameters are the straight joint's impedance, length and propagation coefficient. These parame- ters are extracted by fitting the simulated and measured wave- forms. The optimized parameters are $\mathrm{Zj}=37.0 \Omega, \mathrm{lj}=1.00 \mathrm{~m}$ and $\gamma \mathrm{j}=\gamma \mathrm{c}$. The modeled reflection is shown in Figure 14b.

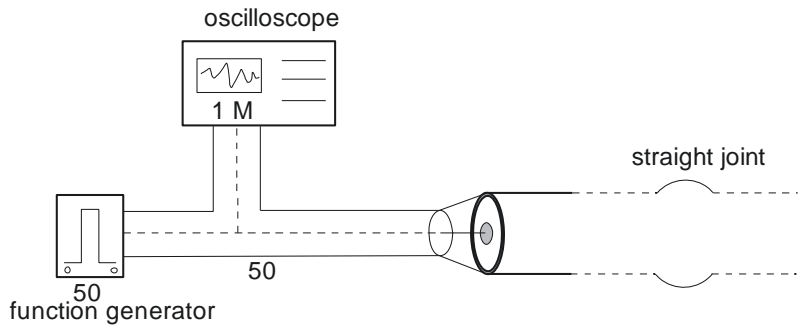

(a) Field measurement set up for power cable joint model verification

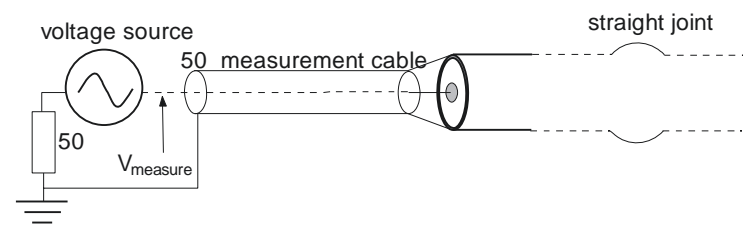

(b) Simulation set up for field measurement

Figure 13. illustation of field measurement and simulation

Besides the cascaded transmission line model, lumped parameter model is also simulated, though the result is not shown here. Comparable results can be obtained as the cascaded transmission line model. The optimized parameters for the lumped model are $\mathrm{Lj}=0.10 \mu \mathrm{H}, \mathrm{Cj}$ $=0.30 \mathrm{pF}$ and $\mathrm{Rj}=1.5 \Omega$. The lumped model is also valid here because the frequency components at the joint refection are within megahertz range. The slight mismatch between the 
Yan Li, et al.

simulation and the measure-ment for the reflection from the adaptor might be because of the adaptor calibration procedure error [7].

\section{Conclusion}

This paper proposes a lumped model and a cascaded transmission line model to described PD signal propagation/reflection through power cable joint. The lumped model is suitable for the frequency range $300 \mathrm{kHz}$ to $10 \mathrm{MHz}$. The transmission line model can cover frequencies up to $800 \mathrm{MHz}$. However, the frequency range may depend on the cable joint's parameters and measurement device and method.

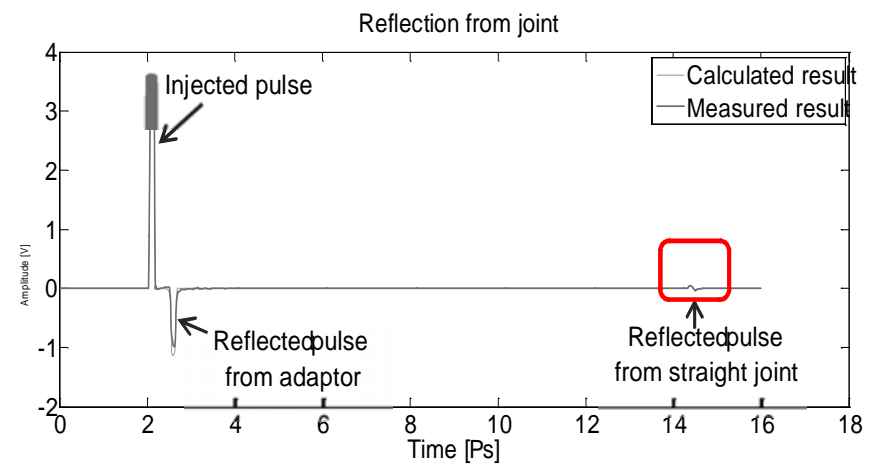

a. Comparison of the whole reflection pattern

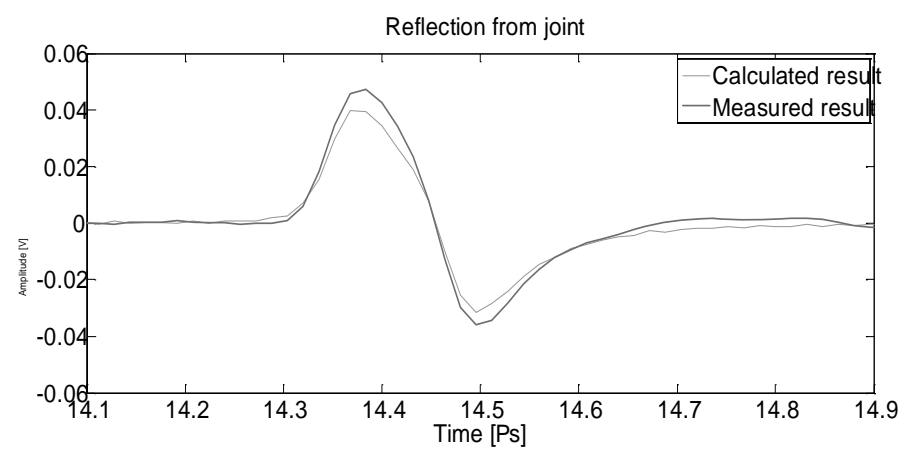

b. Comparison of the reflection from straight joint

Figure 14. Comparison between the measured reflection and the modeled reflection,

The joint models are developed based on a measurement of a $10 \mathrm{kV}$ 3-core PILC straight joint and the transmission line model is successfully used to model the reflection for a $150 \mathrm{kV}$ single core XLPE cable.

The transmission line model also covers the frequency range of the lumped parameter model. Therefore the transmission line model is preferred. However, depending on the application, the lumped parameter model can also be applied if the maximum frequency range is limited .

\section{Acknowledgment}

The authors would like to thank DNV KEMA Energy and Sustainability for their financial support. 


\section{References}

[1] P. C. J. M. van der Wielen, J. Veen, P. A. A. F. Wouters, and E. F. Steennis, “On-line partial discharge detection of mv cables with defect localisation (pdol) based on two time synchronised sensors," in $18^{\text {th }}$ International Conference and Exhibition on Electricity Distribution, 2005. CIRED 2005, June 2005, pp. 1-5.

[2] P. van der Wielen and E. Steennis, "Experiences with continuous condition monitoring of in-service mv cable connections,” in Power Systems Conference and Exposition, 2009. PSCE '09. IEEE/PES, March 2009, pp. 1-8.

[3] M. Michel, "Innovative asset management and targeted investments using on-line partial discharge monitoring \& mapping techniques,” in Proc. 19th Int. Conf. Electricity Distrib. (CIRED), Vienna, Austria, May 2007.

[4] M. Sadiku, Elements of electromagnetics, ser. Oxford series in electrical and computer engineering. Oxford University Press, 2007.

[5] P. Wagenaars, P. Wouters, P. van der Wielen, and E. Steennis, "Propa-gation of pd pulses through ring-main-units and substations," in IEEE 9th International Conference on the Properties and Applications of Dielectric Materials, 2009. ICPADM 2009, July 2009, pp. 441-444.

[6] P. Wagenaars, "Integration of online partial discharge monitoring and defect location in medium-voltage cable networks," Ph.D. dissertation, Eindhoven University of Technology, 2010.

[7] P. Wagenaars, P. Wouters, P. van der Wielen, and E. Steennis, "Measurement of transmission line parameters of three-core power cables with common earth screen," Science, Measurement Technology, IET, vol. 4,no. 3, pp. 146-155, May 2010.

[8] S. Gargari, P. Wouters, P. van der Wielen, and E. Steennis, "Partial discharge parameters to evaluate the insulation condition of on-line located defects in medium voltage cable networks," IEEE Transactions on Dielectrics and Electrical Insulation, vol. 18, no. 3, pp. 868-877, June 2011.

[9] Y. Garcia-Colon, "Power cable on-line diagnosis using partial discharges ultra wide band techniques,” in 2001 Large Engineering Systems Conference on Power Engineering, 2001. LESCOPE '01, 2001, pp. 201-205.

[10] Y. Tian, P. Lewin, and A. Davies, "Comparison of on-line partial discharge detection methods for hv cable joints,” IEEE Transactions on Dielectrics and Electrical Insulation, vol. 9, no. 4, pp. 604-615, Aug 2002.

[11] S. J. Orfanidis, Electromagnetic Waves and Antennas. Rutgers University, 2010.

[12] K. Gupta, R. Garg, and R. Chadha, Computer-Aided Design of Microwave Circuits, ser. Artech House microwave library. Artech, 1981.

[13] W. Eisenstadt and Y. Eo, "S-parameter-based ic interconnect transmission line characterization," IEEE Transactions on Components, Hybrids,and Manufacturing Technology, vol. 15, no. 4, pp. 483-490, Aug 1992.

[14] P. van der Wielen, "On-line detection and location of partial discharges in mediumvoltage power cables,” Ph.D. dissertation, Eindhoven University of Technology, 2005.

[15] R. Papazyan, P. Pettersson, H. Edin, R. Eriksson, and U. Gafvert, "Extraction of high frequency power cable characteristics from s-parameter measurements," IEEE Transactions on Dielectrics and Electrical Insulation, vol. 11, no. 3, pp. 461-470, Jun 2004.

[16] N. Marcuvitz, Waveguide Handbook, ser. IEE Electromagnetic Waves. McGraw-Hill, 1951. 


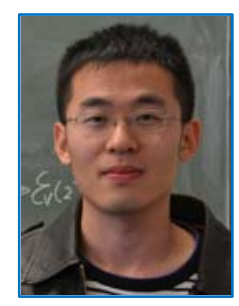

Yan LI was born in Hebei, China, 1985. He studied Electrical Engineering at Zhejiang University, Hangzhou, China, until 2008, from which he received the Bachelor degree. He finished his master study in the Electrical Energy Systems (EES) group at the Technical University of Eindhoven, Eindhoven, The Netherlands in 2010. Now, he is pursuing the PH.D. degree on medium voltage power cable diagnostics techniques in the same group.

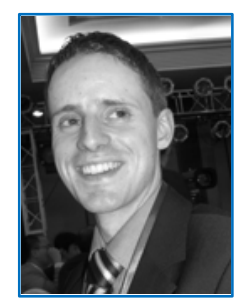

Paul Wagenaars (M’06) was born in The Netherlands in 1981. He received the M.Sc. degree in electrical engineering and the Ph.D. degree from the Eindhoven University of Technology, Eindhoven, The Netherlands, in 2004 and 2010, respectively. His Ph.D. research was on online partial-discharge monitoring of medium-voltage cable systems. After receiving the Ph.D. degree, he joined KEMA as a specialist on power cable technology.

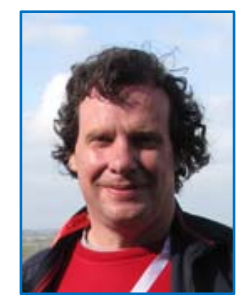

Peter A. A. F. Wouters was born in Eindhoven, The Netherlands, on 9 June, 1957. He studied physics at the Utrecht University (UU), Utrecht, The Netherlands, until 1984, from which he received the Ph.D. degree for a study on elementary electronic transitions between metal surfaces and low energetic (multiple) charged ions in 1989. In 1990, he joined the Electrical Energy Systems (EES) group at the Technical University of Eindhoven, as research associate. His research interests include partial discharge techniques, vacuum insulation, and LF electromagnetic field screening. Currently he is assistant professor in the field of diagnostic techniques in HV systems.

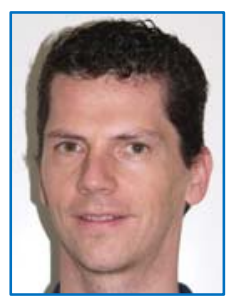

Peter C. J. M. van der Wielen (S93-M01) studied Electrical Engineering at the Eindhoven University of Technology (EUT), where he was also member of the board and many organizing committees of the IEEE Student Branch Eindhoven. He received his M.Sc. degree in 2000. After that he started doing research on power cable diagnostics at both the Electrical Power Systems group at this university and KEMA (now called DNV KEMA) in the Netherlands. In 2005 he received his Ph.D. degree for his study on on-line monitoring of PDs in medium voltage power cables, which evolved into a system now known as DNV KEMA Smart Cable Guard. Since then he has worked, first as a technical specialist, later as a consultant, on power cables and asset management at DNV KEMA. Subjects in the field of work are: power cable consultancy in general, asset management, power cable diagnostics, development of new measuring techniques \& diagnostic methods, remaining life estimations, smart diagnostics and failure analysis. Furthermore, he is one of the lecturers of the various DNV KEMA Power Cable Courses and the Condition and Remaining Lifetime Assessment Course. 


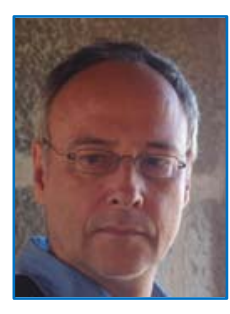

E. Fred Steennis joined KEMA, Arnhem, The Netherlands, in 1982, after his education at the Technical University in Eindhoven, Eindhoven, The Netherlands, where he studied degradation mechanisms in energy cables for the Dutch utilities. It was on this subject that he received the doctors thesis from the Technical University in Delft, Delft, The Netherlands, in 1989. In and outside the Netherlands, he is a consultant on energy cables and is the author and a teacher of the KEMA course on Power Cables. At KEMA, he is the Technical Manager of the Department of High-Voltage Testing and Diagnostics. Since 1999, he is a part-time Professor at the Technical University in Eindhoven, where he teaches and studies diagnostics for power cables. He received the Hidde Nijland award for his contributions in 1991. After that, his experience on degradation mechanisms and related test methods both in the field and the laboratory was further enhanced. Based on this expertise, he became the Dutch representative with the Cigré Study Committee on HighVoltage Cables. He is also a member of various international Working Groups. 